\title{
ClustVarLV: An R Package for the Clustering of Variables Around Latent Variables
}

\author{
by Evelyne Vigneau, Mingkun Chen and El Mostafa Qannari
}

\begin{abstract}
The clustering of variables is a strategy for deciphering the underlying structure of a data set. Adopting an exploratory data analysis point of view, the Clustering of Variables around Latent Variables (CLV) approach has been proposed by Vigneau and Qannari (2003). Based on a family of optimization criteria, the CLV approach is adaptable to many situations. In particular, constraints may be introduced in order to take account of additional information about the observations and/or the variables. In this paper, the CLV method is depicted and the R package ClustVarLV including a set of functions developed so far within this framework is introduced. Considering successively different types of situations, the underlying CLV criteria are detailed and the various functions of the package are illustrated using real case studies.
\end{abstract}

\section{Introduction}

For the clustering of observations, a large number of packages and functions are available within the R environment. Besides the base package stats and the recommended package cluster (Maechler et al., 2015), about one hundred R packages have been listed in the CRAN Task View: Cluster Analysis and Finite Mixture Models (Leisch and Grün, 2015). This is representative of the huge number of applications in which the user is interested in making groups of similar cases, instances, subjects, ..., i.e., in clustering of the observations, in order to exhibit a typology within the population under study. The number of $R$ packages, or $R$ functions, specifically dedicated to the clustering of variables is much smaller.

As a matter of fact, clustering methods (e.g., hierarchical clustering or k-means clustering) are almost always introduced in standard text books using the Euclidean distance between a set of points or observations. Thus, it is not so easy to imagine situations in which defining clusters of variables makes sense. Would it be interesting, in an opinion survey, to identify, a posteriori, groups of questions, and not only clusters of people? The answer to this question is yes, particularly if the number of questions or items is large. Indeed, by merging connected questions, it is possible to identify latent traits, and, as a by-product, improve the interpretation of the outcomes of the subsequent analyses. In another domain, the recent progress in biotechnology enables us to acquire high-dimensional data on a few number of individuals. For instance, in proteomics or metabolomics, recent high-throughput technologies can gauge the abundance of thousands of proteins or metabolites simultaneously. In this context, identifying groups of redundant features appears to be a straightforward strategy in order to reduce the dimensionality of the data set. Based on DNA microarray data, gene clustering is not a new issue. It has usually been addressed using hierarchical clustering algorithms based on similarity indices between each pair of genes defined by their linear correlation coefficient, the absolute value or the squared value of the linear correlation coefficient (see, among others, Eisen et al. 1998; Hastie et al. 2000; Park et al. 2007; Tolosi and Lengauer 2011). We can also mention some specific methods for gene clustering such as the diametrical clustering algorithm of Dhillon et al. (2003) or a clustering method based on canonical correlations proposed by Bühlmann et al. (2013). However, to the best of our knowledge, there is no implementation of these methods in R.

We introduce the ClustVarLV package (Vigneau and Chen, 2015) for variable clustering based on the Clustering of Variables around Latent Variables (CLV) approach (Vigneau and Qannari, 2003). The CLV approach shares several features with the already mentioned approaches of Dhillon et al. (2003) and Bühlmann et al. (2013), as well as with the clustering approach of Enki et al. (2013) for constructing interpretable principal components. It is also worth mentioning that the Valuer's procedure available in SAS (Sarle, 1990) has some common features with the CLV functions of the ClustVarLV package. All these methods are more or less connected to linear factor analysis. They could be viewed as empirical descriptive methods, unlike model-based approaches such as the likelihood linkage analysis proposed by Kojadinovic (2010) for the clustering of continuous variables. Let us note that there is a similar R package, ClustOfVar (Chavent et al., 2013), which proposed an implementation of some of the algorithms described in Vigneau and Qannari (2003). However the ClustOfVar package does not have the same functionalities as the ClustVarLV package. The comparison of these two related packages will be more detailed in a subsequent section.

Other interesting packages for clustering can also be cited: clere (Yengo and Canoui, 2014) for 

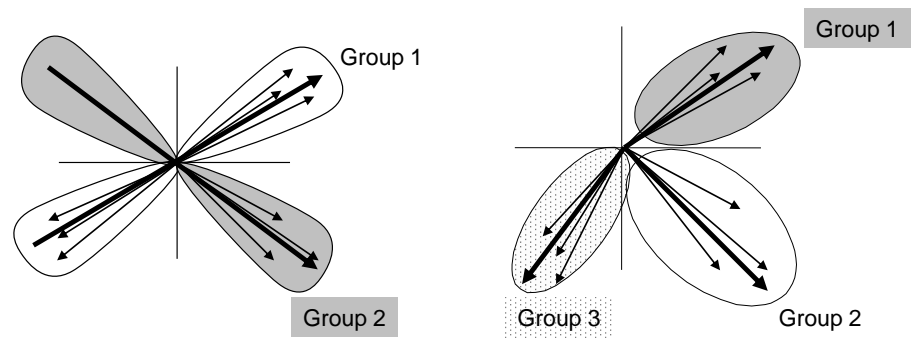

Figure 1: The two possible situations in CLV. On the left side: directional groups (positively and negatively highly correlated variables). On the right side: local groups (positively highly correlated variables). Arrows indicate variables and bold arrows indicate latent components associated with the various groups.

simultaneous variables clustering and regression; biclust (Kaiser et al., 2015) which provides several algorithms to find biclusters in two-dimensional data; pvclust (Suzuki and Shimodaira, 2014) which performs hierarchical cluster analysis and automatically computes $p$-values for all clusters in the hierarchy. This latter package considers the clustering of the columns of a data matrix (for instance, DNA microarray data) and computes (by default) the correlation coefficients between the columns to be clustered. Similarly, the function varclus() in the Hmisc (Harrell Jr et al., 2015) package can be used for performing a hierarchical cluster analysis of variables, using the Hoeffding D statistic, the squared Pearson or Spearman correlations, or the proportion of observations for which two variables are both positive as similarity measures. For pvclust and the function varclus() in package Hmisc, the clustering is done by the hclust () function.

In the following sections, the objective and principle of the CLV approach will be introduced in a comprehensive manner. The main functions of the ClustVarLV package for the implementation of the method will be listed. Next, different situations, associated with various forms of the CLV criterion, will be discussed and illustrated. The first setting will be the case of directional groups of variables for data dimension reduction and the identification of simple structures. Another one will be to identify clusters of variables taking account of an external information.

\section{Synthetic presentation of the CLV method}

In order to investigate the structure of a multivariate dataset, Principal Components Analysis (PCA) is usually used to find the main directions of variation. This can be followed by a rotation technique such as Varimax, Quadrimax,... (Jolliffe, 2002) in order to improve the interpretability to the principal components. The CLV approach is an alternative strategy of analysis whereby the correlated variables are lumped together and, within each cluster, a latent (synthetic) variable is exhibited. This latent variable is defined as a linear combination of only the variables belonging to the corresponding cluster. From this standpoint, CLV has the same objective as the Sparse Principal Component Analysis (Zou et al., 2006) which aims at producing modified principal components with sparse loadings.

The CLV approach (Vigneau and Qannari, 2003) is based on the maximization of a set of criteria which reflect the linear link, in each cluster, between the variables in this cluster and the associated latent variable. These criteria are related to the types of links between the observed and the latent variables that are of interest to the users, as illustrated in Figure 1.

- The first case (left hand panel in Figure 1) is to define directional groups, so that the observed variables that are merged together are as much as possible related to the group latent variable, no matter whether their correlation coefficients are positive or negative. In this case, the link between the observed and the latent variables are evaluated by means of the squared correlation coefficient between the variables, and the criterion considered for maximization is:

$$
T=\sum_{k=1}^{K} \sum_{j=1}^{p} \delta_{k j} \operatorname{cov}^{2} r\left(\mathbf{x}_{j}, \mathbf{c}_{k}\right) \text { with } \operatorname{var}\left(\mathbf{c}_{k}\right)=1
$$

where $\mathbf{x}_{j}(j=1, \ldots, p)$ are the $p$ variables to be clustered. These variables are assumed to be centered. In Equation (1), $K$ is the number of clusters of variables, denoted $G_{1}, G_{2}, \ldots, G_{K} ; c_{k}$ $(k=1, \ldots, K)$ is the latent variable associated with cluster $G_{k}$ and $\delta_{k j}$ reflects a crisp membership, with $\delta_{k j}=1$ if the $j$ th variable belongs to cluster $G_{k}$ and $\delta_{k j}=0$, otherwise. 
- The second case (right hand panel in Figure 1) is to define local groups for which each variable shows a positive correlation with their associated latent variable. This case entails that negative correlation coefficients imply disagreement. Therefore, the CLV criterion is based on the correlation coefficient and the criterion to be maximized is:

$$
S=\sum_{k=1}^{K} \sum_{j=1}^{p} \delta_{k j} \operatorname{cov}\left(\mathbf{x}_{j}, \mathbf{c}_{k}\right) \text { with } \operatorname{var}\left(\mathbf{c}_{k}\right)=1
$$

with the same notations as for Equation (1).

Moreover, as will be illustrated in Section "Clustering of variables with external information" the CLV criteria given in Equations (1) or (2) could be slightly modified, by introducing a constraint on the latent variables, in order to take account of additional information on the variables to be clustered.

It is worth noting that the well known VARCLUS procedure (Sarle, 1990), implemented in the SAS/STAT software, also offers these two options. However, in VARCLUS, no optimization criterion for the determination of the groups of variables is clearly set up. Moreover, this method of analysis consists of a rather complicated divisive hierarchical procedure.

From a practical standpoint, the CLV approach is based on a partitioning algorithm, described in Vigneau and Qannari (2003), akin to the k-means algorithm. However, this partitioning algorithm requires, on the one hand, the choice of the number $K$ of clusters and, on the other hand, the initialization of the iterative process. To address these issues, our recommendation is to start by performing a hierarchical cluster analysis, with aggregating rules detailed in Vigneau and Qannari (2003). The first interest is to set up a dendrogram and a graph showing the evolution of the aggregation criterion between two successive partitions. This should help the user choosing the appropriate number of clusters. The second interest is that the clusters from the hierarchical analysis give reasonable initial partitions for performing the partitioning algorithm. This process of running a partitioning algorithm using the outcomes of the hierarchical clustering as starting point is called consolidation in the French literature ( Lebart et al. 2000; Warms-Petit et al. 2010).

\section{Overview of the functions in the ClustVarLV package}

The list of the functions in the ClustVarLV package, that the users can call, is given in Table 1. The two main functions for the implementation of the CLV algorithms are CLV() and CLV_kmeans().

The CLV() function performs an agglomerative hierarchical algorithm followed by a consolidation step performed on the highest levels of the hierarchy. The number of solutions considered for the consolidation can be chosen by the user (parameter nmax, equal to 20 by default). The consolidation is based on an alternated optimization algorithm, i.e., a k-means partitioning procedure, which is initialized by cutting the dendrogram at the required level. Alternatively, the user may choose to use the CLV_kmeans () function which is typically a partitioning algorithm for clustering the variables into a given number, $K$, of clusters. It involves either repeated random initializations or an initial partition of the variables supplied by the user. This second function may be useful when the number of variables is larger than a thousand because in this case the hierarchical procedure is likely to be time consuming (this point will be addressed in Section "The CLV () and CLV_kmeans() functions"). When the number of variables does not exceed several hundred, the dendrogram which can be drawn from the output of the $\operatorname{CLV}($ ) function provides a useful tool for choosing an appropriate number, $K$, for the size of the partition of variables.

The two functions, $\operatorname{CLV}()$ and $\operatorname{CLV} \_$kmeans(), include a key parameter, which has to be provided by the user, with the data matrix. This parameter, called method, indicates the type of groups that are sought: method = "directional" or method $=1$ for directional groups and method = "local" or method = 2 for local groups (Figure 1). These functions make it possible to cluster the variables of the data matrix (argument $X$ ) considered alone, or by taking account of external information available on the observations (argument $X r$ ) or external information available for the variables themselves (argument $\mathrm{Xu}$ ). A third "CLV" function has been included in the ClustVarLV package: It is the LCLV function which can be used when external information is available for both the observations and the variables (see Section "Clustering of variables with directional groups" for more details).

The other functions in the ClustVarLV package (version 1.4.1) are mainly utility and accessor functions providing additional outputs useful for the interpretation of the clustering results. Their usage will be illustrated with various case studies that will be discussed hereinafter. 


\begin{tabular}{ll}
\hline Functions & Description \\
\hline & \multicolumn{1}{c}{ “Clustering" functions } \\
\hline $\begin{array}{l}\text { CLV } \\
\text { CLV_kmeans } \\
\text { LCLV }\end{array}$ & $\begin{array}{l}\text { Hierarchical clustering of variables with consolidation } \\
\text { Kmeans algorithm for the clustering of variables } \\
\text { L-CLV for L-shaped data }\end{array}$ \\
\hline & \multicolumn{1}{c}{ Methods for 'clv' objects } \\
\hline $\begin{array}{l}\text { plot } \\
\text { print }\end{array}$ & Graphical representation of the CLV clustering stages \\
\hline & Print the CLV results \\
\hline $\begin{array}{l}\text { plot } \\
\text { print }\end{array}$ & Methods for 'lclv' objects \\
\hline & Praphical representation of the LCLV clustering stages \\
\hline $\begin{array}{l}\text { summary } \\
\text { plot_var } \\
\text { get_partition the LCV results } \\
\text { get_comp } \\
\text { get_load }\end{array}$ & $\begin{array}{l}\text { Method providing the description of the clusters of variables } \\
\text { To get the clusters of variables and their group membership }\end{array}$ \\
\hline & To get the latent variables associated with each cluster \\
\hline $\begin{array}{l}\text { stand_quali } \\
\text { data_biplot }\end{array}$ & $\begin{array}{l}\text { Standardization of the qualitative variables } \\
\text { Biplot for the dataset }\end{array}$ \\
\hline
\end{tabular}

Table 1: List of the functions in the ClustVarLV package.

\section{Clustering of variables with directional groups}

As indicated above, when the user chooses method = "directional" in the CLV() or CLV_kmeans () function, the criterion considered for optimization is the criterion $T$ defined in Equation (1).

It can be shown (see for instance Vigneau and Qannari 2003) that when the maximum of the criterion $T$ is reached, the latent variable $\mathbf{c}_{k}$, in cluster $G_{k}$, is the first normalized principal component of matrix $\mathbf{X}_{k}$, the dataset formed of the variables belonging to $G_{k}$. Thus, the optimal value of $T^{(K)}$, for a partition into $K$ groups, is the sum of the largest eigenvalues respectively associated with the variance-covariance matrices $\frac{1}{n} \mathbf{X}_{k}^{\prime} \mathbf{X}_{k}$, with $k=1, \ldots, K$. The ratio between $T^{(K)}$ and $T^{(p)}$ provides the percentage of the total variance explained by the KCLV latent variables. Even if the $K$ CLV latent variables, which are not necessarily orthogonal, cannot take account of as much total variance as the $K$ first principal components, they may be more relevant for deciphering the underlying structure of the variables than the first principal components. Moreover, they are likely to be more easily interpretable. Enki et al. (2013) have also addressed the issue of identifying more interpretable principal components and proposed a procedure which bears some similarities with the CLV method.

\section{First illustrative example: Identification of block structure, and underlying latent com- ponents, into a set of variables}

We consider data from a French Research Project (AUPALESENS, 2010-2013) dealing with foodbehavior and nutritional status of elderly people. More precisely, we selected the psychological behavior items, which are part of a large questionnaire submitted to 559 subjects. As a matter of fact, the 31 psychological items were organized into five blocks, each aiming to describe a given behavioral characteristic: emotional eating $(E)$ with six items, external eating $(X)$ with five items, restricted eating $(\mathrm{R})$ with five items, pleasure for food $(\mathrm{P})$ with five items, and self esteem $(\mathrm{S})$ with ten items. Detailed description and analysis of the emotional, external and restricted eating items for this study are available in Bailly et al. (2012).

The CLV() function was performed on the data matrix, $\mathbf{X}$, which merges the 31 psychological items, using the following code:

R> library ("ClustVarLV")

R> data("AUPA_psycho", package = "ClustVarLV") 

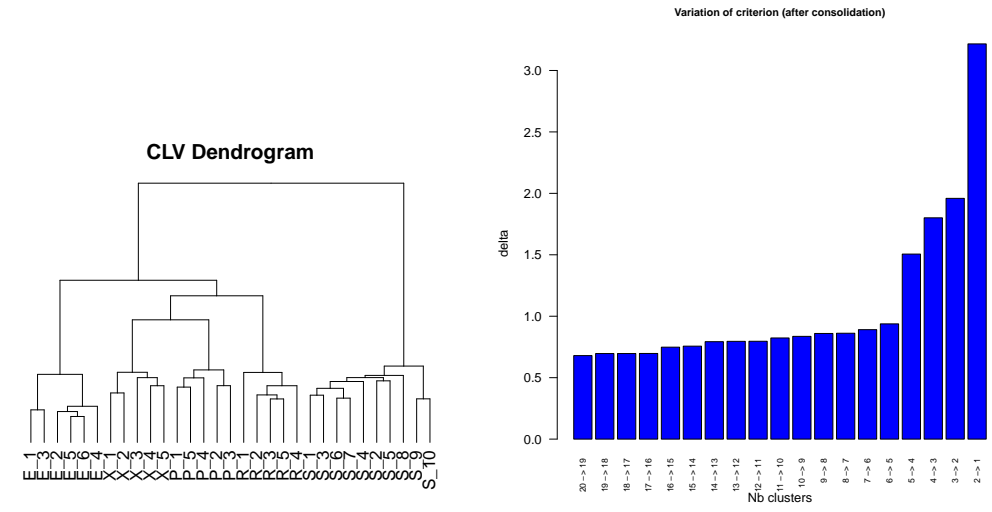

Figure 2: Graphs obtained by the clustering into directional groups of the psychological variables. On the left side, the dendrogram of the hierarchical clustering stage; on the right side, the variation of the clustering criterion after consolidation of the partitions by means of the partitioning algorithm.

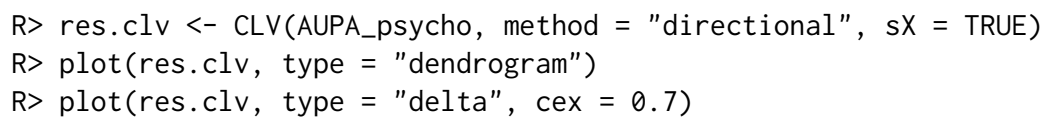

The dendrogram and the graph showing the variation of the clustering criterion when passing from a partition into $K$ clusters to a partition into $(K-1)$ clusters $\left(\right.$ Delta $\left.=T^{(K)}-T^{(K-1)}\right)$ are shown in Figure 2. From the graph of Delta, it can be observed that the criterion clearly jumps when passing from five to four clusters. This means that the loss in homogeneity of the clusters is important with four clusters and that a partition into five clusters should be retained. The partition into $K=5$ groups, available with get_partition (res.clv, $K=5$ ), perfectly retrieved the five blocks of psychological traits.

The summary method for 'clv' objects provides a description of the clusters:

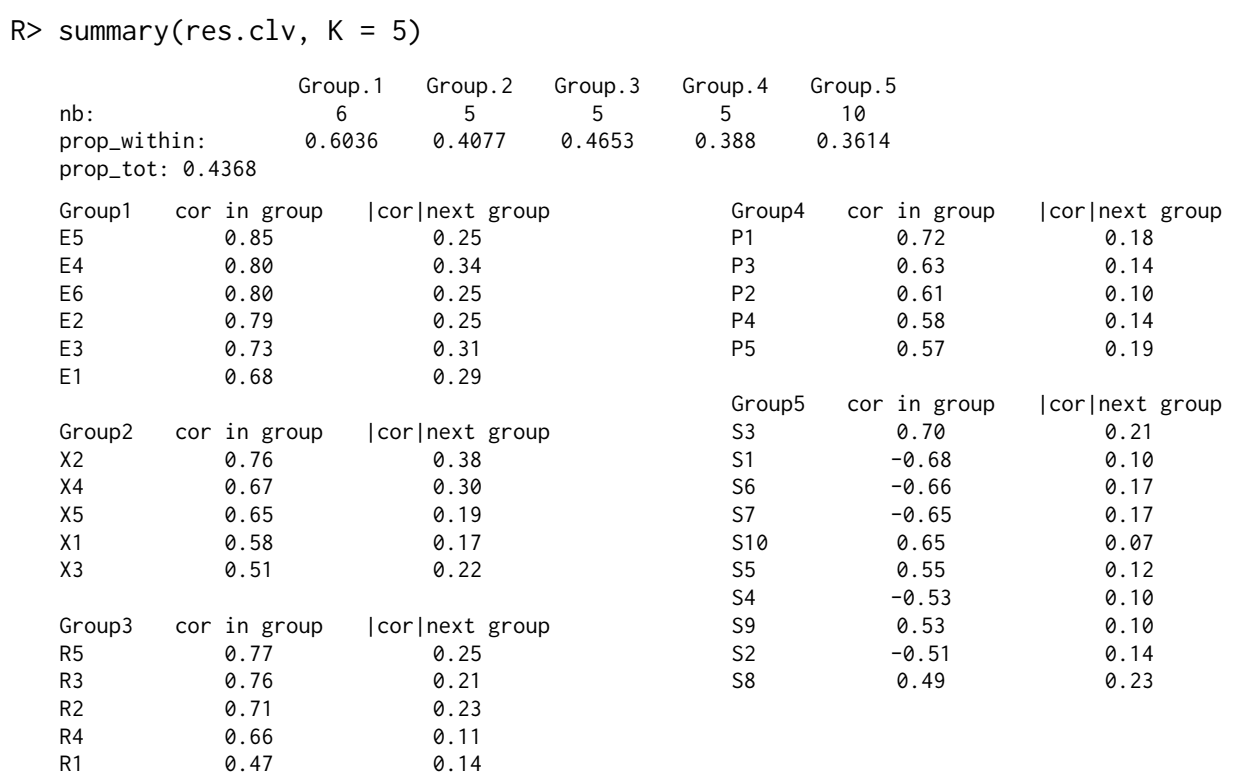

The homogeneity values within each cluster, assessed by the percentages of the total variance of the variables belonging to the cluster explained by the associated latent variable, are $60.4 \%, 40.8 \%$, $46.5 \%, 38.8 \%, 36.1 \%$ respectively (the Cronbach's alphas are $0.87,0.63,0.71,0.60$, and 0.80 , respectively). Furthermore, the five group latent variables make it possible to explain $43.7 \%$ of the total variance of all the $p=31$ observed variables. For each variable in a cluster, its correlation coefficient with its own group latent variable and its correlation coefficient with the next nearest group latent variable are also given. Each item is highly correlated with its group latent variable.

Compared with the standardized PCA of $\mathbf{X}$, five principal components (PCs) are required for retrieving $45.1 \%$ of the total variance, whereas four PCs account for $40.5 \%$ of the total variance. Moreover, it turned out that the interpretation of the first five PCs was rather difficult. If we consider 
all the loadings larger than 0.3 , in absolute value, the first $P C, P C 1$, seems to be associated with all the items " $\mathrm{E}^{\prime}, \mathrm{X} 2, \mathrm{X} 3, \mathrm{R} 2$ and S8; PC2 is related to $\mathrm{P} 1$ and all the items " $\mathrm{S}$ " except S8, PC3 to R1 only, PC4 to X4, R3, R4, R5 and P3, and PC5 to X1 and X5. It is known that rotation (by means of orthogonal or oblique transformations) may enhance the interpretation of the factors. In this case study, using a Varimax transformation, the five rotated PCs can be associated with one of the predefined blocks of items. However, the rotated principal components make it possible to retrieve the "true" structure if, and only if, the correct number of dimensions for the subspace of rotation is selected. This may be an impediment since the determination of the appropriate number of components is a tricky problem. In the case study at hand, various rules (Jolliffe, 2002) led to two, four or eight PCs. By contrast, the variation of the CLV criterion performs well for identifying the correct number of groups.

In another domain (i.e., the Health sector), Lovaglio (2011) pointed out that, within the Structural Equation Modeling framework, the first step which consists of building the measurement models could be based on the CLV technique. He showed that, considering a formative way, the subset of variables obtained by means of $\operatorname{CLV}()$ led to a better recovery of the original configuration, followed by VARCLUS based on PCA. This was far from being the case with the selection of variables on the basis of the outcomes of PCA or PCA with Varimax rotation.

\section{Second illustrative example: Clustering of quantitative and qualitative variables}

Chavent et al. (2012) proposed an R package, named ClustOfVar, which aims at clustering variables, with the benefit of allowing the introduction of quantitative variables, qualitative variables or a mix of those variables. The approach is based on a homogeneity criterion which extends the CLV criterion (Eq.1). More precisely, the correlation ratio (between groups variance to total variance ratio) of each qualitative variable and the latent variable in a cluster are included in the criterion in addition to the squared correlation coefficients used for the quantitative variables. In practice, for defining the partition of the variables and the latent variables within each cluster, the algorithms described in Chavent et al. (2012) are the same as those given in Vigneau and Qannari (2003) and Vigneau et al. (2006), with a small variation: The latent variables are derived from a PCAMIX model (Saporta, 1990; Kiers, 1991; Pagès, 2004) instead of a PCA model.

The strategy of clustering quantitative and qualitative variables raises the following question: Is it better to cluster qualitative variables along with the quantitative variables or to break down each qualitative variable into its categories and include these categories in a clustering approach such as CLV?

To answer this question, let us consider the dataset 'wine' provided in various packages (for instance, ClustOfVar, FactoMineR, Husson et al. (2015)). 21 french wines of Val of Loire are described by 29 sensory descriptors scored by wine professionals. Two nominal variables are also provided: the label of the origin (with three categories: "Saumur", "Bourgueuil" and "Chinon") and the nature of the soil (with four categories: "Reference", "Env.1", "Env.2" and "Env.4"). The design of these two nominal variables is however not well-balanced. Chavent et al. (2012) considered only 27 quantitative variables (all the sensory descriptors except those regarding the global evaluation) and included the two qualitative variables. From the dendrogram obtained with the function hclustvar(), they retained six clusters. The summary of the partition into six clusters is shown below:

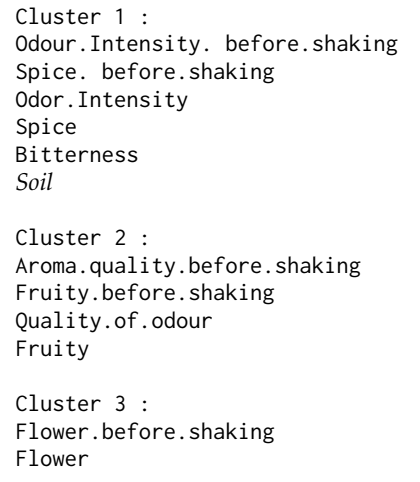

$\begin{array}{cl}\text { squared loading } & \text { Cluster 4: } \\ 0.76 & \text { Visual.intensity } \\ 0.62 & \text { Nuance } \\ 0.67 & \text { Surface.feeling } \\ 0.54 & \text { Aroma.intensity } \\ 0.66 & \text { Aroma.persistency } \\ 0.78 & \text { Attack.intensity } \\ & \text { Astringency } \\ \text { squared loading } & \text { Alcohol } \\ 0.78 & \text { Intensity } \\ 0.85 & \text { Cluster } 5: \\ 0.79 & \text { Plante } \\ 0.91 & \text { Aroma.quality } \\ \text { squared loading } & \text { Acidity } \\ 0.87 & \text { Balance } \\ 0.87 & \text { Smooth } \\ & \text { Harmony } \\ & \text { Cluster } 6: \\ & \text { Phenolic } \\ & \text { Label }\end{array}$

squared loading
0.86
0.84
0.90
0.75
0.86
0.77
0.79
0.68
0.87

squared loading
0.75
0.84
0.22
0.94
0.92
0.87
squared loading
0.8
0.8

The factor "Soil" was merged in the Cluster 1 with variables related to spicy sensation and the odor intensity. Its correlation ratio with the latent variable of this cluster is 0.78 (which corresponds to a $F$-ratio $=19.73$ with a $p$-value $=9 \mathrm{E}-6$ ). The factor "Label" was merged in the cluster 6 with the quantitative descriptor "Phenolic". The correlation ratio of "Label" with the latent variable of its 


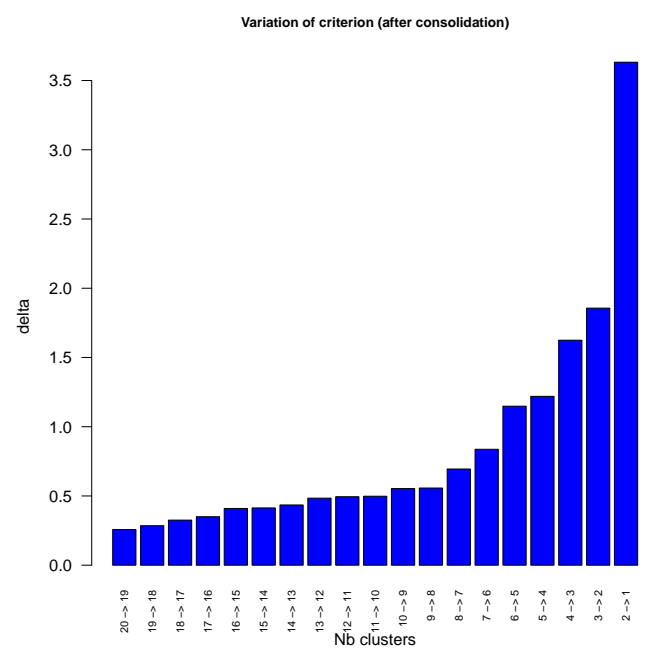

Figure 3: Graph showing the evolution of the aggregation criterion after consolidation.

cluster is $0.80(F$-ratio $=36.02, p$-value $=5 \mathrm{E}-7)$.

In the ClustVarLV package, we propose to take account of the qualitative information, in addition to quantitative variables, by breaking down each qualitative variable into a matrix of indicators $(G$, say) of size $n \times M$, where $M$ is the number of categories of the qualitative variable at hand. In the same vein as Multiple Correspondence Analysis (Saporta, 1990), we propose to standardize the matrix G. This leads us to the matrix $\tilde{\mathbf{G}}=\mathbf{G D}^{-1 / 2}$ where $\mathbf{D}$ is the diagonal matrix containing the relative frequency of each category. The utility function stand_quali() in ClustVarLV allows us to get the matrix $\tilde{\mathbf{G}}$. Thereafter, the matrix submitted to the $\operatorname{CLV}()$ function is simply the concatenation of the standardized matrix of the quantitative variables and all the standardized blocks associated with each qualitative variables. The following code was used:

R> library("ClustVarLV")

$\mathrm{R}>$ data("wine", package $=$ "FactoMineR")

$\mathrm{R}>\mathrm{X}$.quanti <- wine[, 3:29]

$\mathrm{R}>\mathrm{X}$.quali $<-$ wine $[, 1: 2]$

R> Xbig <- $\operatorname{cbind}(\operatorname{scale}(X . q u a n t i)$, stand_quali(X.quali))

$\mathrm{R}>$ resclv <- CLV(Xbig, method = "directional", $\mathrm{sX}=$ FALSE)

R> plot (resclv, "delta")

From the graph showing the evolution of the aggregation criterion (Figure 3), two, four, six or even eight clusters could be retained.

The partition into six clusters is described as follows:

$\mathrm{R}>\operatorname{summary}(\mathrm{resclv}, \mathrm{K}=6)$

\begin{tabular}{|c|c|c|c|c|c|}
\hline Group1 & cor in group & |cor|next group & Group4 & cor in group & |cor|next group \\
\hline Odour. Intensity. before.shaking & 0.87 & 0.63 & Surface.feeling & 0.95 & 0.80 \\
\hline Soil.Env.4 & 0.86 & 0.43 & Intensity & 0.94 & 0.82 \\
\hline Odour. Intensity & 0.82 & 0.69 & Visual.intensity & 0.93 & 0.64 \\
\hline Spice. before.shaking & 0.80 & 0.32 & Aroma.persistency & 0.93 & 0.76 \\
\hline Bitterness & 0.80 & 0.49 & Nuance & 0.92 & 0.63 \\
\hline \multirow[t]{2}{*}{ Spice } & 0.73 & 0.40 & Astringency & 0.89 & 0.70 \\
\hline & & & Attack.intensity & 0.88 & 0.74 \\
\hline Group2 & cor in group & |cor|next group & Aroma.intensity & 0.87 & 0.78 \\
\hline Aroma.quality & 0.93 & 0.64 & Alcohol & 0.83 & 0.59 \\
\hline Balance & 0.93 & 0.68 & & & \\
\hline Smooth & 0.92 & 0.77 & Group5 & cor in group & |cor|next group \\
\hline Quality.Odour & 0.90 & 0.71 & Phenolic & 0.89 & 0.42 \\
\hline Harmony & 0.90 & 0.87 & Label.Bourgueuil & -0.86 & 0.30 \\
\hline Aroma.quality.before. shaking & 0.81 & 0.74 & Label.Saumur & 0.77 & 0.40 \\
\hline Plante & -0.78 & 0.42 & & & \\
\hline Fruity.before.shaking & 0.77 & 0.58 & Group6 & cor in group & |cor|next group \\
\hline \multirow[t]{2}{*}{ Soil.Reference } & 0.70 & 0.46 & Acidity & 0.89 & 0.30 \\
\hline & & & Soil.Env.2 & 0.69 & 0.35 \\
\hline Group3 & cor in group & |cor|next group & Soil.Env.1 & -0.68 & 0.37 \\
\hline Flower before.shaking & 0.93 & 0.44 & Label.Chinon & 0.63 & 0.22 \\
\hline Flower & 0.93 & 0.35 & & & \\
\hline
\end{tabular}

It turns out that both functions, i.e., hclustvar () in ClustOfVar (hierarchical algorithm) and CLV() in ClustVarLV (hierarchical algorithm followed by a partitioning procedure), led to similar results for 
the sensory descriptors.

The first group (Group 1) is related to the intensity of the odor with spicy notes, to which is associated the "Env.4" for the "Soil" factor, whereas it was globally "Soil" using hclustvar(). If we compare the correlation ratio of the qualitative variable "Soil" with its cluster latent variable using hclustvar() (i.e., 0.78), and the squared correlation coefficient of the category "Soil.Env.4" with its cluster latent variable using CLV() (i.e., 0.74), we can conclude that the contribution of the three other "Soil" categories to the correlation ratio is very small. This finding can easily be confirmed by means of a one-way ANOVA between the latent variable in the first cluster and the factor "Soil". Additionally, it can be shown that the correlation ratio $\left(R^{2}\right)$ of a qualitative variable with respect to a quantitative variable ( $b f x$, say) is equal to a weighted sum of the squared correlation coefficients of the indicators of its categories, given in $\tilde{G}$, with the quantitative variable, namely:

$$
R^{2}=\sum_{m=1}^{M}\left(1-f_{m}\right) \operatorname{cor}^{2}\left(\mathbf{g}_{m}, \mathbf{x}\right),
$$

where $\mathbf{g}_{m}$ is the indicator vector for the category $m$ and $f_{m}$ is the relative frequency. It follows that, the contribution of "Soil.Env.4" to the global $R^{2}$ of "Soil" in the first cluster found with hclustvar() is $85.4 \%$. Thus, it appears that it is because of the specific nature of the soil in "Env.4" that the wines have a more intense odor and a more bitter flavor than the other wines.

The second group of attributes (Group 2) is related to the overall quality of the wines and it seems, from the results of $\operatorname{CLV}()$, that the type "Reference" of the soil is likely to favor this quality. This was not observed with hclustvar() (see Cluster 5 in the summary of the partition into six clusters obtained with hclustvar()) because the qualitative variable "Soil" was globally associated with the Cluster 1 .

Regarding the fifth groups of attributes (Group 5), the interpretation of the Phenolic flavor of some wines could be refined. If the "Label" was associated with the Phenolic attribute using hclustvar() (Cluster 6), the outputs of the CLV() function show that type "Saumur" was slightly more "Phenolic" than the type "Bourgeuil", whereas type "Chinon" (in Group 6) seems to have acid notes (but caution should be taken in this interpretation because of the small number of observations for "Chinon"). Nevertheless, it could be emphasized that the soil of "Env.2" is likely to give more acidity, unlike "Env.1". Finally let us notice that the acidity attribute was merged in the Cluster 5 obtained with hclustvar() but its squared loading to the latent variable of this cluster was relatively small.

\section{Clustering of variables for local groups}

In some specific situations, a negative correlation between two variables is considered as a disagreement. Therefore, these variables should not be lumped together in the same group.

Consider for instance the case of preference (or acceptability) studies in which consumers are asked to give a liking score for a set of products. For these data, the consumers play the role of variables whereas the products are the observations. The aim is to identify segments of consumers having similar preferences. This means positively correlated vectors of preference. In this situation, local groups are sought (illustrated in the right side of Figure 1) and the parameter method = "local" is to be used with the clustering functions of the ClustVarLV package. A case study developed in this context is available in Vigneau et al. (2001).

In other contexts, as in Near-Infrared spectroscopy or ${ }^{1} \mathrm{H}$ NMR spectroscopy, the CLV approach with local groups can be used for a first examination of the spectral data. Jacob et al. (2013) showed that this approach may help identifying spectral ranges and matching them with known compounds.

Technically, the identification of local groups of variables is performed in the CLV approach by the maximization of the criterion $S$ given in Equation (2). As a result, it is easy to show that the maximal value is obtained, for a given number $K$ of clusters, when each latent variable, $\mathbf{c}_{k}$, is proportional to the centroid variable $\overline{\mathbf{x}}_{k}$ of the variables in the cluster $G_{k}$.

\section{Third illustrative example: Application to the segmentation of a panel of consumers}

In order to illustrate the use of the ClustVarLV functions for the definition of local groups, let us consider the dataset 'apples_sh' available in the package (Daillant-Spinnler et al., 1996). Two types of information were collected: On the one hand, the sensory characterization, given by a trained panel, of 12 apple varieties from the Southern Hemisphere and, on the other hand, the liking scores, given by 60 consumers, for these varieties. We will consider the segmentation of the panel of consumers using the $\operatorname{CLV}()$ function with the option method = "local":

R> library ("ClustVarLV")

R> data("apples_sh", package = "ClustVarLV") 

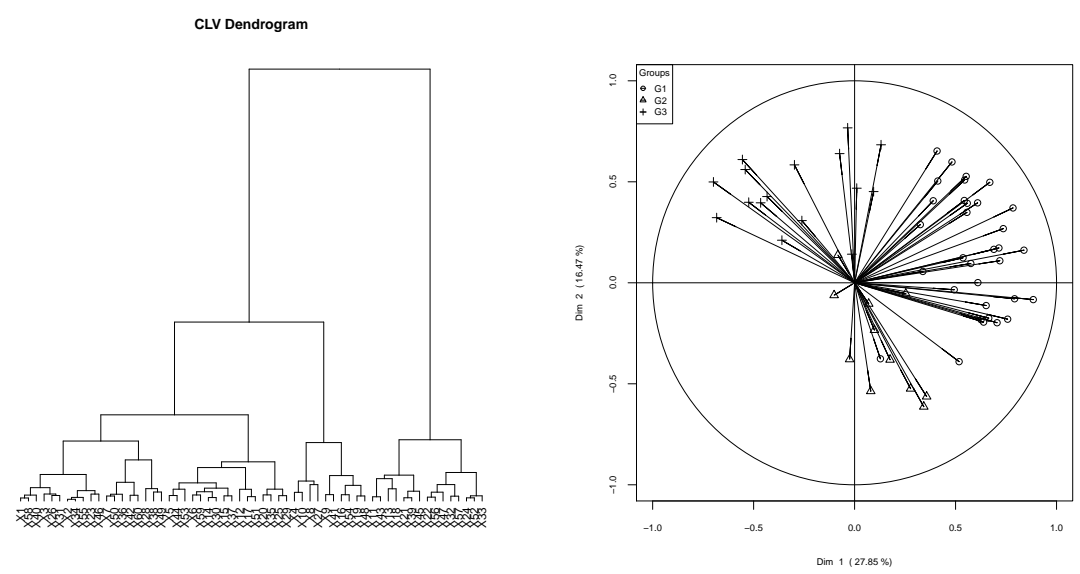

Figure 4: Segmentation of the panel of consumers for the apples case study. On the left side, the dendrogram of the hierarchical clustering; on the right side, the mapping of the consumers on the basis of the two first principal components, with group membership identification.

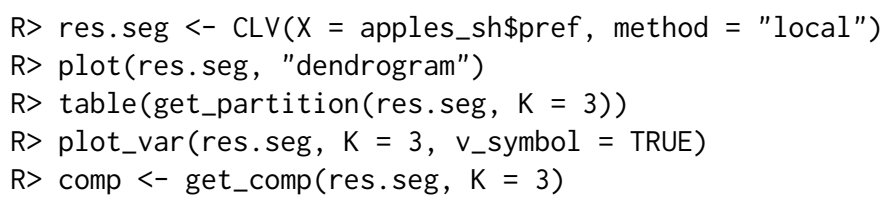

The dendrogram from $\operatorname{CLV}()$ given in the left side of Figure 4 suggests to retain three segments. These segments merged together 33, 11 and 16 consumers, respectively (after consolidation of the solution obtained by cutting the dendrogram at the chosen level). The plot_var() companion function makes it possible to show the group membership of each variable on a two dimensional subspace. The plot produced by this function (right side of Figure 4) is grounded on a PCA loading plot. By default, the two first principal components are considered, but the user may modify this option. In the previous code, the option ' $v$ _symbol' is set to TRUE in order to produce a figure readable in black and white. Without this option, color graphs will be produced, with or without the labels of the variables. In addition, the group latent variables may be extracted with the function get_comp(). They provide the preference profiles of the 12 apple varieties in the various consumer segments.

\section{Clustering of variables with external information}

The CLV approach has also been extended to the case where external information is available. The clustering of variables is achieved while constraining the group latent variables to be linear combinations of external variables.

\section{Clustering with external information collected on the observations}

Suppose that, in addition to the variables to be clustered, the observations are described by a second block of variables, $\mathbf{X r}$ ( $r$ stands for additional information collected on the rows of the core matrix $\mathbf{X}$ ) as in Figure 5. Both CLV criteria (Equations 1 and 2) can be used with the additional constraint that:

$$
\mathbf{c}_{k}=\operatorname{Xr} \mathbf{a}_{k} \text { with } \mathbf{a}_{k}^{\prime} \mathbf{a}_{k}=1
$$

for each latent variable $\mathbf{c}_{k}$ with $k=1, \ldots, K$.

It can be shown (Vigneau and Qannari, 2003) that the solutions of the optimization problems are obtained when $\mathbf{c}_{k}$ is the first component of a Partial Least Squares (PLS) regression of the group matrix $\mathbf{X}_{k}$ on the external matrix $\mathbf{X}_{\mathbf{r}}$, in the case of directional groups, or the first component of a PLS regression of the centroid variable $\overline{\mathbf{x}}_{k}$ on the external matrix $\mathbf{X r}$, in the case of local groups.

External preference mapping is a domain in which the CLV approach with additional information on the observations has been successfully applied (Vigneau and Qannari, 2002). In addition to clustering the consumers according to the similarity of their preference scores as it was illustrated in the third illustrative example, the aim is also to segment the consumers while explaining their preferences by means of the sensory characteristics of the products. Thus, the segmentation and the modeling of the main directions of preference may be achieved simultaneously. If we consider again 
the 'apples_sh' dataset, two matrices are available: 'apples_sh\$pref', the preference scores of the consumers, and 'apples_sh\$senso', the sensory characterization of the 12 apple varieties using 43 sensory attributes. The CLV() function includes parameters for taking account of such external block of information. Namely:

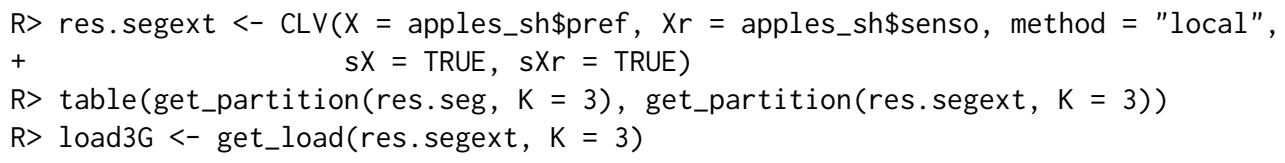

For a solution with three clusters, it turns out that the segments previously defined have been rearranged in order to take account of the sensory attributes of the apples. The loadings $\mathbf{a}_{k}$ (for $k=1,2,3)$ of the sensory descriptors, which can be extracted using the utility function get_load(), made it possible to explain the difference in preference in each segment.

\section{Clustering with additional information on the variables}

When additional information is available on the variables, the CLV approach has also been adapted in order to take this information into account in the clustering process.

For instance, let us consider the problem of the clustering of spectral variables. Typically, a spectrometer (Near Infrared or a Nuclear Magnetic Resonance spectrometer) makes it possible to collect thousands of measurements at different spectral variables (wavelengths or chemical shifts). This leads to a large amount of information with high level of redundancy since close spectral points convey more or less the same information. Instead of trimming off close spectral points, the clustering of variables is a more effective way of identifying automatically spectral ranges associated with the same functional chemical groups (Vigneau et al., 2005). However, the fact that the variables correspond to successive wavelengths was not taken into account with the previous criteria given in Equation 1, or Equation 2. One can expect that adding information on the spectral structure of the variables can improve the quality of the clusters of variables, in the sense that variables within the same spectral range are more likely to be lumped together. The additional information to be considered in such a situation is related to the spectral proximity between the variables.

We denote by $\mathbf{Z}$, the matrix of the additional information on the variables. The rows in $\mathbf{Z}$ are matched with the columns of the matrix $\mathbf{X}$. The CLV approach is performed by combining, in each cluster of variables, the $\mathrm{X}$ - and the Z-information. Namely, for a given cluster $G_{k}$, a new matrix $\mathbf{P}_{k}$ is defined by:

$$
\mathbf{P}_{k}=\mathbf{X}_{k} \mathbf{Z}_{k}
$$

where $\mathbf{X}_{k}$ is the sub-matrix of $\mathbf{X}$ formed by the $p_{k}$ variables belonging to $G_{k}$, and similarly, $\mathbf{Z}_{k}$ is a sub-matrix of $\mathbf{Z}$ which involves only these $p_{k}$ variables. Thus, $\mathbf{P}_{k}$ can be viewed as a weighted version of $\mathbf{X}_{k}$, or as an interaction matrix between the $X$ - and $Z$-information estimated within $G_{k}$. The nature of $\mathbf{Z}$, as well as the pretreatment applied, lead to one or the other point of view. The CLV criteria have been modified so that the latent variable in a cluster is a linear combination of the associated $\mathbf{P}_{k}$ matrix. If we denote by $\mathbf{t}_{k}$ the latent variable in the cluster $G_{k}$, the objective is either to maximize

$$
T^{Z}=\sum_{k=1}^{K} \sum_{j=1}^{p} \delta_{k j} \operatorname{cov}^{2}\left(\mathbf{x}_{j}, \mathbf{t}_{k}\right)
$$

or

$$
S^{Z}=\sum_{k=1}^{K} \sum_{j=1}^{p} \delta_{k j} \operatorname{cov}\left(\mathbf{x}_{j}, \mathbf{t}_{k}\right)
$$

with the constraints that:

and

$$
\mathbf{t}_{k}=\mathbf{P}_{k} \mathbf{u}_{k} / \operatorname{trace}\left(\mathbf{P}_{k}^{\prime} \mathbf{P}_{k}\right)
$$

$$
\mathbf{u}_{k}^{\prime} \mathbf{u}_{k}=1
$$

The parameter $\mathrm{Xu}$ in the $\mathrm{CLV}($ ) function makes it possible to take account of the external information on the variables. A typical line of code in this case may be as:

$\mathrm{R}>$ resclv $<-\operatorname{CLV}(X=X, X u=Z$, method = "local", $s X=F A L S E)$

\section{Clustering with additional information on the observations and the variables}

When external informations on observations and variables are available, $\mathbf{X}, \mathbf{X r}$ and $\mathbf{Z}$ are associated either by their rows or by their columns, so that the three blocks of data may be arranged in the form 


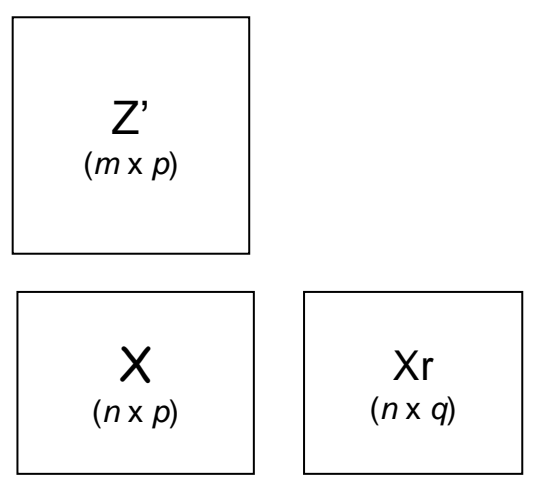

Figure 5: L-shaped data.

of an L (Figure 5). Therefore, the acronym $L-C L V$ has been adopted and the LCLV() function included in the package ClustVarLV has been developed for this case.

The L-CLV approach directly stems from the previous extensions of the CLV approach. It consists in the maximization, in each cluster $k$ (with $k=1, \ldots, K$ ) of the covariance between a pair of latent variables, $\mathbf{c}_{k}$ and $\mathbf{t}_{k} \cdot \mathbf{c}_{k}$ is a linear combination of the co-variables measured on the observations, $\mathbf{X r}_{\mathbf{r}}$ and $\mathbf{t}_{k}$ is a linear combination of the $\mathbf{P}_{k}$ variables (defined in the previous section). The criterion to be maximized is:

$$
\tilde{T}=\sum_{k=1}^{K} \operatorname{cov}\left(\mathbf{c}_{\mathrm{k}}, \mathbf{t}_{\mathrm{k}}\right) \text { with } \mathbf{c}_{\mathrm{k}}=\mathbf{X r}_{\mathbf{r}} \mathbf{a}_{\mathrm{k}}, \mathbf{t}_{\mathrm{k}}=\mathbf{P}_{\mathrm{k}} \mathbf{u}_{\mathrm{k}}=\mathbf{X}_{\mathrm{k}} \mathbf{Z}_{\mathrm{k}} \mathbf{u}_{\mathrm{k}} \text { and } \mathbf{a}_{\mathrm{k}}^{\prime} \mathbf{a}_{\mathrm{k}}=1, \mathbf{u}_{\mathrm{k}}^{\prime} \mathbf{u}_{\mathrm{k}}=1
$$

or alternatively,

$$
\tilde{T}=\sum_{k=1}^{K} \mathbf{u}_{k}^{\prime} \mathbf{Z}_{k}^{\prime} \mathbf{X}_{k}^{\prime} \mathbf{X r} \mathbf{a}_{k}
$$

From the expression in Equation 9, it turns out that L-CLV bears strong similarities with the so-called L-PLS method (Martens et al., 2005). The main difference lies in the fact that L-CLV involves a clustering process and that a specific matrix mixing the $\mathbf{X}, \mathbf{X r}$ and $\mathbf{Z}$ informations is considered and updated in each cluster.

Interested readers are referred to Vigneau et al. (2011) and Vigneau et al. (2014) for further details and an illustration of the procedure for the segmentation of a panel of consumers, according to their likings ( $\mathbf{X}$ ), interpretable in terms of socio-demographic and behavioral parameters (given in $\mathbf{Z}$ ) and in relation with the sensory key-drivers (in $\mathbf{X r}$ ). For such case studies the $\operatorname{LCLV}($ ) function has been used with the following code (default options used):

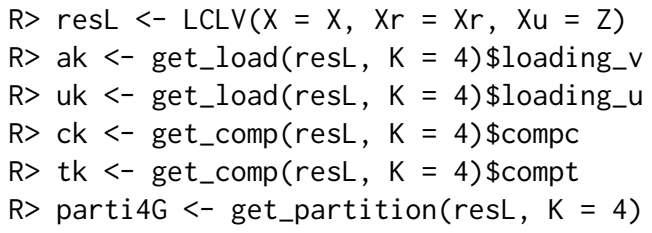

The function get_load() allows one to extract, for a given number of clusters $K$, the loadings $\mathbf{a}_{k}$ and the loadings $\mathbf{u}_{k}$. This makes it possible to interpret the results in the light of the external informations. The latent variables $\boldsymbol{c}_{k}$ and $\mathbf{t}_{k}$ (for $k=1, \ldots, K$ ) are also available using the function get_comp() and the cluster membership of the variables are provided with the function get_partition().

\section{Technical considerations}

\section{The CLV() and CLV_kmeans() functions}

The CLV() function was described for the clustering of variables, for local or directional groups, when external information is taken into account or not. This function involves two stages, a hierarchical algorithm followed by a non-hierarchical (or partitioning) algorithm. As a matter of fact, the hierarchical algorithm provides, at a given level, $h$, an optimal partition conditionally on the partition obtained at the previous level, $h-1$. The partitioning algorithm starts with the partition obtained by cutting 


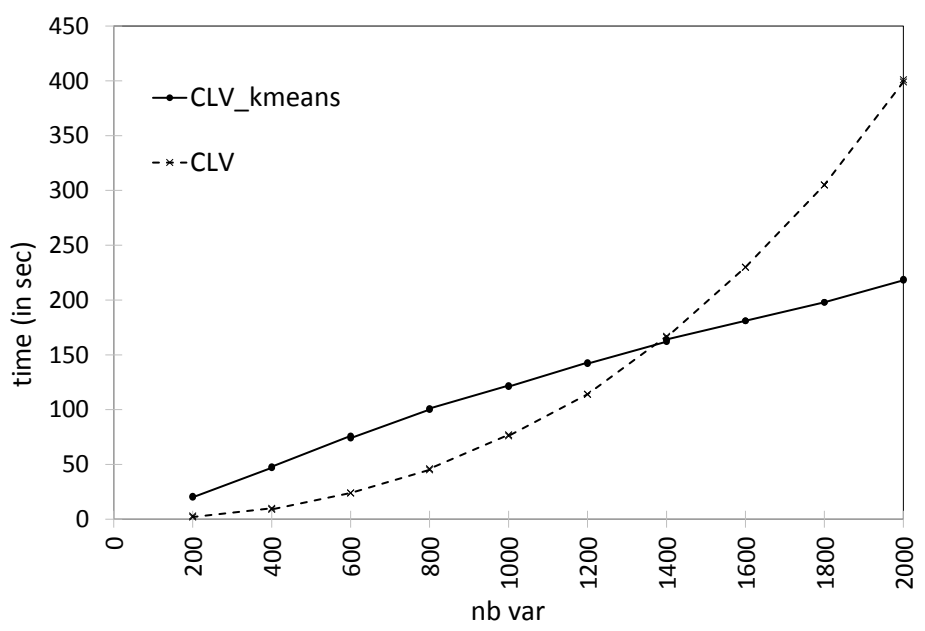

Figure 6: Comparison of the processing time with $\operatorname{CLV}()$ and $\operatorname{CLVkmeans}()$ as a function of the number of variables (the other parameters of the experiment being fixed).

the dendrogram at a given level (say, $h$ ) and an alternating optimization scheme is used until the convergence of the criterion to be maximized. The number of iterations before convergence is given in the list of the results (e.g., resclv\$tabres[, "iter"]). This second stage is called consolidation stage. By default, the consolidation is performed for the last twenty levels of the hierarchy, i.e., for $K=1$ to $K=20$.

However, when the number of variables is large, the hierarchical algorithm may be time consuming. For this reason, the CLV_kmeans() function was added to the package ClustVarLV. This function has the same parameters and options as the $\operatorname{CLV}()$ function, but performs only the partitioning stage. In this case, the number of clusters, $K$, should be given as an input parameter. For the initialization of the iterative algorithm, the user may suggest a partition used as a starting point, or, may ask that random initializations of the algorithm are repeatedly performed. The number of repetitions in case of random initializations is stated by the user (argument nstart).

Figure 6 shows that the time required for the CLV_kmeans() function increases approximately linearly with the number of variables. Let us notice that in this experiment, there were twenty observations, the nstart parameter was fixed to 50, and the CLV_kmeans () function was used iteratively twenty times, by varying the number of clusters from $K=1$ to $K=20$. In comparison, the relationship between the time required for the $\operatorname{CLV}($ ) function (consolidation done for $K=1$ to $K=20$ ) and the number of variables looks like a power function. As can be observed (Figure 6), when the number of variables was about 1400, the processing time was comparable for both procedures. When the number of variables was larger, as it is often the case when dealing with -omics data, the CLV_kmeans() function (used for partitions into one cluster until twenty clusters) provides a faster implementation. However, for reasonable number of variables to cluster, the $\operatorname{CLV}()$ function appears preferable. This is not only because $\operatorname{CLV}()$ is relatively fast in this case, but also because it provides a graph of the evolution of the aggregation criterion which is helpful for choosing the number of clusters.

\section{The ClustOfVar and ClustVarLV packages}

As stated above, both packages, ClustOfVar and ClustVarLV, are devoted to the cluster analysis of variables. They both draw from the same theoretical background (Vigneau and Qannari, 2003). We emphasize hereinafter some differences of these two packages.

In the first place, it seems that ClustVarLV is less time consuming than ClustOfVar. To illustrate this aspect, we considered a large dataset, named "Colon", which is available in the plsgenomics package (Boulesteix et al., 2015). It concerns the gene expression of 2000 genes for 62 samples from the microarray experiments of Colon tissue samples of Alon et al. (1999). As shown below, the running time was less than 7 minutes for the $\operatorname{CLV}()$ function, whereas the hclustvar() of the ClustOfVar required more than an hour and a half. The performance of $\operatorname{CLV}()$ over hclustvar() can be partly explained by the fact that ClustVarLV is interfaced with $\mathrm{C++}$ blocks of code thanks to the Rcpp package (Eddelbuettel and François, 2011; Eddelbuettel, 2013).

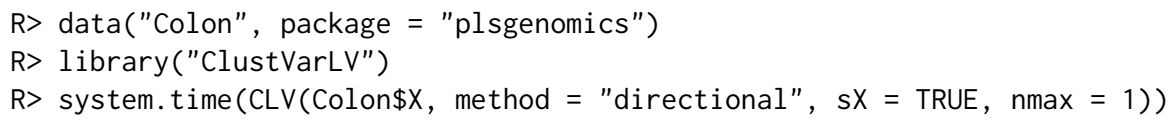


$\begin{array}{lll}\text { user } & \text { system } & \text { elapsed } \\ 385.30 & 7.60 & 392.95\end{array}$

R> library("ClustofVar")

R> system.time(hclustvar (Colon $\$ X)$ )

user system elapsed

$4926.37 \quad 15.57 \quad 4942.44$

We also indicated that the feature in ClustOfVar that is generally put forward is the possibility to cluster both quantitative and qualitative variables. We have stressed through the 'wine' dataset the limitation of clustering together quantitative and qualitative variables and we advocated breaking down the qualitative variables into the indicator variables associated with its categories. It is also worth mentioning that ClustVarLV covers a much wider scope than ClustOfVar as it makes it possible:

(i) to cluster variables according to local (method = "local") or directional groups (method = "directional"), this latter option being the only possibility offered by ClustOfVar;

(ii) to perform a cluster analysis on non standardized ( $\mathrm{SX}=$ FALSE) or standardized variables $(s X=$ TRUE), whereas ClustOfVar systematically standardizes the variables;

(iii) to cluster the variables taking into account external information on the observations and/or the variables.

\section{Concluding remarks}

The R package ClustVarLV contains the functions CLV, CLV_kmeans and LCLV related to the CLV approach, which can be used with or without external information. Additional functions have also been included in order to extract different types of results or to enhance the interpretation of the outcomes. A vignette is included in the package documentation (web link: ClustVarLV) and provides some basic examples for running the main functions of the ClustVarLV package.

Several developments of the CLV approach are under investigation and will be implemented in the forthcoming updates of the ClustVarLV package. The "cleaning up" of the variables which do not have a clear assignment to their current cluster (noise variables, for instance) is one of the issues that we are investigating. Another interesting topic is the clustering of variables with the aim of explaining a given response variable as described in Chen and Vigneau (in press).

\section{Bibliography}

U. Alon, N. Barkai, D. Notterman, K. Gish, S. Ybarra, D. Mack, and A. Levine. Broad patterns of gene expression revealed by clustering analysis of tumor and normal colon tissues probed by oligonucleotide arrays. Proceedings of the National Academy of Sciences of the United States of America, 96(12):6745-6750, 1999. [p145]

N. Bailly, I. Maitre, M. Amand, C. Hervé, and D. Alaphilippe. The Dutch eating behaviour questionnaire (DEBQ). Assessment of eating behaviour in an aging French population. Appetite, 59(3): 853-858, 2012. [p137]

A.-L. Boulesteix, G. Durif, S. Lambert-Lacroix, J. Peyre, and K. Strimmer. plsgenomics: PLS Analyses for Genomics, 2015. URL https: //CRAN. R-project. org/package=plsgenomics. R package version 1.3-1. [p145]

P. Bühlmann, P. Rütimann, S. van de Geer, and C.-H. Zhang. Correlated variables in regression: Clustering and sparse estimation. Journal of Statistical Planning and Inference, 143(11):1835_1858, 2013. [p134]

M. Chavent, V. Kuentz-Simonet, B. Liquet, and J. Saracco. ClustOfVar: An R package for the clustering of variables. Journal of Statistical Software, 50(13):1-16, 2012. URL http: //www. jstatsoft. org/v50/ i13/. [p139]

M. Chavent, V. Kuentz, B. Liquet, and J. Saracco. ClustOfVar: Clustering of Variables, 2013. URL https://CRAN. R-project.org/package=ClustofVar. R package version 0.8. [p134]

M. Chen and E. Vigneau. Supervised clustering of variables. Advanced in Data Analysis and Classification, in press. doi: 10.1007/s11634-014-0191-5. [p146] 
B. Daillant-Spinnler, H. MacFie, P. Beyts, and D. Hedderley. Relationships between perceived sensory properties and major preference directions of 12 varieties of apples from the Southern Hemisphere. Food Quality and Preference, 7(2):113-126, 1996. [p141]

I. S. Dhillon, E. M. Marcotte, and U. Roshan. Diametrical clustering for identifying anti-correlated gene clusters. Bioinformatics, 19(13):1612-1619, 2003. [p134]

D. Eddelbuettel. Seamless R and C++ Integration with Rcpp. Springer, New York, 2013. [p145]

D. Eddelbuettel and R. François. Rcpp: Seamless R and C++ integration. Journal of Statistical Software, 40(8):1-18, 2011. URL http: //www. jstatsoft.org/v40/i08/. [p145]

M. B. Eisen, P. T. Spellman, P. O. Brown, and D. Botstein. Cluster analysis and display of genome-wide expression patterns. Proceedings of the National Academy of Sciences of the United States of America, 95 (25):14863-14868, 1998. [p134]

D. G. Enki, N. T. Trendafilov, and I. T. Jolliffe. A clustering approach to interpretable principal components. Journal of Applied Statistics, 40(3):583-599, 2013. [p134, 137]

F. E. Harrell Jr, C. Dupont, et al. Hmisc: Harrell Miscellaneous, 2015. URL https: //CRAN. R-project. org/package=Hmisc. R package version 3.17-0. [p135]

T. Hastie, R. Tibshirani, M. B. Eisen, A. Alizadeh, R. Levy, L. Staudt, W. C. Chan, D. Botstein, and P. Brown. 'Gene shaving' as a method for identifying distinct sets of genes with similar expression patterns. Genome Biology, 1(2):1-21, 2000. [p134]

F. Husson, J. Josse, S. Le, and J. Mazet. FactoMineR: Multivariate Exploratory Data Analysis and Data Mining, 2015. URL https://CRAN.R-project.org/package=FactoMineR. R package version 1.31.4. [p139]

D. Jacob, C. Deborde, and A. Moing. An efficient spectra processing method for metabolite identification from 1H-NMR metabolomics data. Analytical and Bioanalytical Chemistry, 405(15):5049-5061, 2013. [p141]

I. Jolliffe. Principal Component Analysis. Springer-Verlag, New York, 2nd edition, 2002. [p135, 139]

S. Kaiser, R. Santamaria, T. Khamiakova, M. Sill, R. Theron, L. Quintales, F. Leisch, and E. De Troyer. biclust: BiCluster Algorithms, 2015. URL https: //CRAN. R-project. org/package=biclust. R package version 1.2.0. [p135]

H. Kiers. Simple structure in component analysis techniques for mixtures of qualitative and quantitative variables. Psychometrika, 56(2):197-212, 1991. [p139]

I. Kojadinovic. Hierarchical clustering of continuous variables based on the empirical copula process and permutation linkages. Computational Statistic and Data Analysis, 54(1):90-108, 2010. [p134]

L. Lebart, A. Morineau, and M. Piron. Statistique exploratoire multidimensionnelle. Dunod, Paris, 3ieme edition, 2000. [p136]

F. Leisch and B. Grün. CRAN task view: Cluster analysis \& finite mixture models, 2015. URL https://CRAN.R-project.org/view=Cluster. Version 2015-07-24. [p134]

P. G. Lovaglio. Model building and estimation strategies for implementing the balanced scorecard in health sector. Quality $\mathcal{E}$ Quantity, 45(1):199-212, 2011. [p139]

M. Maechler, P. Rousseeuw, A. Struyf, and M. Hubert. cluster: "Finding Groups in Data": Cluster Analysis Extended Rousseeuw et al., 2015. URL https://CRAN. R-project.org/package=cluster. R package version 2.0.3. [p134]

H. Martens, E. Anderssen, A. Flatberg, L. H. Gidskehaug, M. Hoy, F. Westad, A. Thybo, and M. Martens. Regression of a matrix on descriptors of both its rows and its columns via latent variables: L-PLSR. Computational Statistics and Data Analysis, 48(1):103-123, 2005. [p144]

J. Pagès. Analyse factorielle de données mixtes. Revue de Statistique Appliquée, 52(4):93-111, 2004. [p139]

M. Y. Park, T. Hastie, and R. Tibshirani. Averaged gene expressions for regression. Biostatistics, 8(2): 212-227, 2007. [p134]

G. Saporta. Simultaneous analysis of qualitative and quantitative data. In Societa Italiana di Statistica, editor, Atti Della XXXV Riunione Scientifica, pages 63-72, 1990. [p139, 140] 
W. Sarle. SAS/STAT User's Guide: The Varclus Procedure. SAS Institute, Inc., Cary, NC, USA, 4th edition, 1990. [p134, 136]

R. Suzuki and H. Shimodaira. pvclust: Hierarchical Clustering with P-Values via Multiscale Bootstrap Resampling, 2014. URL https://CRAN. R-project.org/package=pvclust. R package version 1.3-2. [p135]

L. Tolosi and T. Lengauer. Classification with correlated features: unreliability of feature ranking and solutions. Bioinformatics, 27(14):1986-1994, 2011. [p134]

E. Vigneau and M. Chen. ClustVarLV: Clustering of Variables Around Latent Variables, 2015. URL https: //CRAN.R-project.org/package=ClustVarLV. R package version 1.4.1. [p134]

E. Vigneau and E. Qannari. Clustering of variables around latent components. Communications in Statistics - Simulation and Computation, 32(4):1131-1150, 2003. [p134, 135, 136, 137, 139, 142, 145]

E. Vigneau and E. M. Qannari. Segmentation of consumers taking account of external data: A clustering of variables approach. Food Quality and Preference, 13(7-8):515-521, 2002. [p142]

E. Vigneau, E. M. Qannari, P. H. Punter, and S. Knoops. Segmentation of a panel of consumers using clustering of variables around latent directions of preference. Food Quality and Preference, 12(5-7): 359-363, 2001. [p141]

E. Vigneau, K. Sahmer, E. M. Qannari, and D. Bertrand. Clustering of variables to analyze spectral data. Journal of Chemometrics, 19(3):122-128, 2005. [p143]

E. Vigneau, E. M. Qannari, K. Sahmer, and D. Ladiray. Classification de variables autour de composantes latentes. Revue de Statistique Appliquée, 54(1):27-45, 2006. [p139]

E. Vigneau, I. Endrizzi, and E. Qannari. Finding and explaining clusters of consumers using the CLV approach. Food Quality and Preference, 22(4):705-713, 2011. [p144]

E. Vigneau, M. Charles, and M. Chen. External preference segmentation with additional information on consumers: A case study on apples. Food Quality and Preference, 22(4):83-92, 2014. [p144]

E. Warms-Petit, E. Morignat, M. Artois, and D. Calavas. Unsupervised clustering of wildlife necropsy data for syndromic surveillance. BMC Veterinary Research, 6:56, 2010. URL http: //www. biomedcentral. com:1746-6148/6/56. [p136]

L. Yengo and M. Canoui. clere: Simultaneous Variables Clustering and Regression, 2014. URL https: //CRAN. R-project. org/package=clere. R package version 1.1.2. [p134]

H. Zou, T.Hastie, and R.Tibshirani. Sparse principal component analysis. Journal of Computational and Graphical Statistics, 15(2):262-286, 2006. [p135]

\author{
Evelyne Vigneau \\ Sensometrics and Chemometrics Laboratory \\ National College of Veterinary Medicine, Food Science and Engineering (Oniris) \\ Rue de la Geraudiere, CS 82225 \\ 44322 Nantes Cedex 03 \\ France \\ evelyne.vigneau@oniris-nantes. fr \\ Mingkun Chen \\ Sensometrics and Chemometrics Laboratory \\ National College of Veterinary Medicine, Food Science and Engineering (Oniris) \\ Rue de la Geraudiere, CS 82225 \\ 44322 Nantes Cedex 03 \\ France \\ El Mostafa Qannari \\ Sensometrics and Chemometrics Laboratory \\ National College of Veterinary Medicine, Food Science and Engineering (Oniris) \\ Rue de la Geraudiere, CS 82225 \\ 44322 Nantes Cedex 03 \\ France
}

